\title{
School based education programme to reduce salt intake in children and their families (School-EduSalt): cluster randomised controlled trial
}

\author{
Feng J He, ${ }^{1}$ Yangfeng Wu, 2, 3, 4 Xiang-Xian Feng, ${ }^{5}$ Jun Ma, ${ }^{6}$ Yuan Ma, ${ }^{1}, 2,3$ Haijun Wang, ${ }^{6}$ Jing Zhang, ${ }^{2}$ \\ Jianhui Yuan, ${ }^{5}$ Ching-Ping Lin, ${ }^{2,7}$ Caryl Nowson, ${ }^{8}$ Graham A MacGregor ${ }^{1}$
}

${ }^{1}$ Wolfson Institute of Preventive Medicine, Barts and The London School of Medicine and Dentistry, Queen Mary University of London, London, UK ${ }^{2}$ George Institute for Global Health at Peking University Health Science Center, Beijing, China

${ }^{3}$ Department of Epidemiology and Biostatistics, Peking University School of Public Health, Beiijing, China

${ }^{4}$ Peking University Clinical Research Institute, Beijing, China ${ }^{5}$ Changzhi Medical College, Shanxi, China

Institute of Child and

Adolescent Health, Peking University Health Science

Center, Beijing, China

7 University of Michigan Medical School, Ann Arbor, MI, USA

${ }^{8} \mathrm{School}$ of Exercise and

Nutrition Sciences, Deakin University, Geelong, Australia

Correspondence to:

FHe f.he@qmul.ac.uk

Additional material is published online only. To view please visit the journal online (http:// dx.doi.org/10.1136/BMJ.h770)

Cite this as: $B M J$ J 2015;350:h770 doi=10.1136/bmj.h770

Accepted: 09 January 2015

\section{ABSTRACT}

\section{OBJECTIVE}

To determine whether an education programme targeted at schoolchildren could lower salt intake in children and their families.

DESIGN

Cluster randomised controlled trial, with schools randomly assigned to either the intervention or control group.

\section{SETTING}

28 primary schools in urban Changzhi, northern China.

PARTICIPANTS

279 children in grade 5 of primary school, with mean age of 10.1; 553 adult family members (mean age 43.8). INTERVENTION

Children in the intervention group were educated on the harmful effects of salt and how to reduce salt intake within the schools' usual health education lessons. Children then delivered the salt reduction message to their families. The intervention lasted for one school term (about 3.5 months).

\section{MAIN OUTCOME MEASURES}

The primary outcome was the difference between the groups in the change in salt intake (as measured by 24 hour urinary sodium excretion) from baseline to the end of the trial. The secondary outcome was the difference between the two groups in the change in blood pressure.

\section{WHAT IS ALREADY KNOWN ON THIS TOPIC}

Salt intake is high in China, and salt is added mainly by the consumers. Salt reduction is one of the most cost effective measures to reduce the disease burden related to raised blood pressure

There have been no successful salt reduction programmes in countries where most of the salt in the diet is added by consumers. Furthermore, it is not clear whether an education programme targeted at primary school children could lower salt intake in children and their families

\section{WHAT THIS STUDY ADDS}

A reduction in salt intake can be achieved by integrating salt reduction education modules into primary school curriculums and empowering children to deliver the salt reduction message to their families

This is a novel, feasible, and effective approach to reducing salt intake in a population in which most of the salt in the diet is added by consumers

Our findings suggest that the WHO's target of 30\% reduction in salt intake by 2025 could be achieved in China if the education programme was implemented nationwide and could result in a major reduction in cardiovascular morbidity and mortality

\section{RESULTS}

At baseline, the mean salt intake in children was 7.3 (SE $0.3) \mathrm{g} /$ day in the intervention group and 6.8 (SE 0.3) $\mathrm{g} /$ day in the control group. In adult family members the salt intakes were 12.6 (SE 0.4) and 11.3 (SE 0.4) g/day, respectively. During the study there was a reduction in salt intake in the intervention group, whereas in the control group salt intake increased. The mean effect on salt intake for intervention versus control group was $-1.9 \mathrm{~g} /$ day $(95 \%$ confidence interval -2.6 to $-1.3 \mathrm{~g} /$ day; $\mathrm{P}<0.001)$ in children and $-2.9 \mathrm{~g} /$ day $(-3.7$ to $-2.2 \mathrm{~g} /$ day; $P<0.001)$ in adults. The mean effect on systolic blood pressure was $-0.8 \mathrm{~mm} \mathrm{Hg}$ ( -3.0 to $1.5 \mathrm{~mm} \mathrm{Hg}$; $\mathrm{P}=0.51)$ in children and $-2.3 \mathrm{~mm} \mathrm{Hg}(-4.5$ to $-0.04 \mathrm{~mm}$ $\mathrm{Hg} ; \mathrm{P}<0.05)$ in adults.

\section{CONCLUSIONS}

An education programme delivered to primary school children as part of the usual curriculum is effective in lowering salt intake in children and their families. This offers a novel and important approach to reducing salt intake in a population in which most of the salt in the diet is added by consumers.

TRIAL REGISTRATION

ClinicalTrials.gov NCT01821144.

\section{Introduction}

Cardiovascular disease is the leading cause of death and disability worldwide. About $80 \%$ of deaths from cardiovascular disease occur in developing countries. ${ }^{1}$ Raised blood pressure is a major cause of such disease, accounting for $62 \%$ of strokes and $49 \%$ of cases of ischaemic heart disease. ${ }^{2}$ Dietary salt intake is the main factor that increases blood pressure and is largely responsible for the rise in blood pressure with age. ${ }^{3}{ }^{4}$ There is compelling evidence in adults that a modest reduction in salt intake lowers blood pressure and reduces the risk of cardiovascular disease. ${ }^{4-8}$ Indeed, salt reduction is one of the most cost effective measures to prevent cardiovascular disease in both developed and developing countries. ${ }^{9-11}$ The World Health Organization has recommended salt reduction as one of the top three priority actions to tackle the global crisis in non-communicable disease. ${ }^{12} 13$

Although raised blood pressure and cardiovascular disease typically present in adults, the origins begin in childhood. ${ }^{14} 15$ Accordingly, the greatest long term potential to reduce these conditions is to initiate prevention activities in young people. Such a public heath strategy aimed at preventing or slowing the progression of rising blood pressure altogether would have enormous 
benefits. Several lines of evidence from animal experiments, epidemiological studies, and controlled trials have shown that salt intake plays an important role in regulating blood pressure in children. ${ }^{16} \mathrm{~A}$ lower salt diet starting from childhood could lessen the rise in blood pressure with age and therefore help to prevent the development of high blood pressure and cardiovascular disease later in life.

China is the largest developing country in the world. Raised blood pressure caused by excessive salt consumption is highly prevalent. ${ }^{17}$ The problem is particularly marked in northern China, where salt intake is high in both adults ${ }^{18}$ and children. Unlike in developed countries, the major source of salt in the Chinese diet is salt added by the consumers themselves during food preparation. ${ }^{19} 20$ An important strategy to reduce population salt intake would be to encourage the general public to reduce the amount of salt used at home. To date, no country has demonstrated a successful programme where salt intake has fallen because consumers have been educated to use less salt. Indeed, it is difficult to persuade individuals to change their diet.

The School-EduSalt (School-based Education Programme to Reduce Salt) study aimed to develop a novel approach to lowering salt intake. In China, children have a big influence on the family. Our approach was to educate primary school children about the harmful effects of excessive salt intake and to empower them to educate their families to reduce the amount of salt used at home. We hypothesised that such an education programme could lower salt intake in both children and their families. To test this hypothesis, we carried out a cluster randomised controlled trial in Changzhi, northern China.

\section{Methods}

A detailed description of the methods of the SchoolEduSalt study has been published elsewhere. ${ }^{21}$

\section{Study design and participants}

The study was a cluster randomised controlled trial. Primary schools in urban Changzhi were eligible. We excluded schools in rural areas to avoid contamination from school meals as some children in such schools have lunch and dinner at school because of the long distance from home, whereas in urban schools most children have lunch and dinner at home.

There are 36 primary schools in urban Changzhi. We excluded eight schools: three special Muslim schools, two schools that were far from the study centre, and three schools with headteachers who were not willing to participate. The 28 remaining schools were included.

In primary schools in Changzhi, there are six grades for children aged 7-12. Our study was carried out in grade 5 children (age about 10) only. From each school, we selected one class (if there was more than one) in grade 5. With the headteacher we selected a class whose teacher in charge was willing to collaborate with the researchers.

We used a random number list (created by a researcher who was blind to the identity of the participants) to randomly select children from each class until 10 children were recruited. To be eligible for inclusion in the assessments, children had to eat homemade meals at least three days a week and live not too far from the school $(<3 \mathrm{~km})$. In two schools where the class had fewer than 15 children, we invited all children to take part in the assessments.

From each child's family we invited two adults for assessments. All adults who shared the same meals with the child were eligible. If more than two adults in one family were willing to participate, we selected two of them (one man and one woman) in the order of grandparents, parents, uncles, and aunts.

\section{Randomisation}

Schools (clusters) were randomly assigned (1:1) to either the intervention or the control group. Randomisation was stratified by the location of schools (urban or suburban) and the size of the class. Among all schools in urban Changzhi, the size of the class varied from 14 to 75 children a class. Usually, a larger class size indicates that the school is at the upper level of the league table in terms of exam results.

An independent statistician who was blind to the identity of the schools carried out the randomisation using a computer generated random number system. The randomisation took place after written consents had been obtained and the baseline assessments had completed. The participants, the schoolteachers, and the local investigators who undertook participant recruitment and data collection were therefore unaware of the allocation until the point before the start of the intervention.

\section{Intervention}

Our aim was to reduce salt intake by a minimum of $20 \%{ }^{21}$ To achieve this, we set 50\% reduction as the target, toward which an intervention strategy was developed and implemented..$^{21}$ The intervention duration was one school term (about 3.5 months). Our slogan was "small hands leading big hands, together let's reduce salt." The salt reduction curriculum and materials were developed around the key messages: the harmful effects of salt on health and the salt reduction target; the recommended level of salt intake; and how to reduce salt intake.

Local health educators who were trained by research staff over a four day workshop delivered the salt reduction education programme. A detailed intervention manual and resources were provided. The programme materials consisted of lesson plans, activity worksheets, and homework assignments. The materials were developed around cartoon characters. A detailed description of the education programme is provided in the appendix.

\section{Main components of programme Classroom component}

For the selected classes, the usual health education lessons (a 40 minute lesson, every two weeks; a total of eight lessons in one school term of about 3.5 months) were replaced with lessons on salt reduction. The lessons were delivered to the whole class, despite 
only 10 children being selected for assessments. The lessons included three proactive and interactive lectures, one themed class meeting to share experiences, one class meeting for family participation, and three other activities to assist in salt reduction, such as competitions on writing and illustration, plays among children, and salt reduction knowledge involving both children and adults with family as a unit (see appendix). Schoolteachers assisted in the lessons and coordinated all events. Posters on the harmful effects of salt and how to reduce salt intake were put up in the classroom.

Children were asked to complete the following tasks as homework: emphasise the $50 \%$ salt reduction target and remind the whole family of this target after each lesson; deliver the salt reduction messages, salt reduction methods, and skilful tips to the whole family; and develop a salt reduction action plan for their own family and supervise the actions at home.

\section{Family component}

Children needed to persuade the people who did the cooking to reduce the amount of salt, soy sauce, and bean paste used. Garlic, ginger, and herbs were recommended for enhancing food flavour. We also recommended replacing pickles with fresh vegetables and replacing salted eggs and peanuts with unsalted ones. We encouraged participants to replace the usual salt with a mineral salt that is low in sodium and high in potassium. No special foods or salt substitutes were provided, and the important message was to reduce the total amount of salt used.

Parents were provided with educational materials in the form of a newsletter (see appendix) that covered topics such as salt and its effect on blood pressure and cardiovascular disease, the major sources of salt in the diet, and cooking with reduced salt. The newsletter also had a question and answer column and a family quiz. Homework set for the students was related to the newsletter.

\section{Monitoring of family use of salt}

As more than $75 \%$ of salt in the diet came from salt added during food preparations at home, ${ }^{19}$ we estimated the approximate amount of salt used per family and monitored it every two weeks. Each family was provided with a container, and parents were asked to put all of the salt in their household into the container and use salt from this container only. Children brought the salt container to school every two weeks, and the teachers measured the weight. The amount of salt used by the household for those two weeks was calculated with a computer programme that also provided information on how far the family's salt use was different from the target set (that is, 50\% reduction). The results were communicated back to the families.

We reported the amount of salt used as grams per person per day (that is, the total amount of salt used by the family divided by the number of family members). Each family was also provided with a salt control spoon (2 g salt). Parents were asked to use this spoon if they thought it was necessary to add salt during cooking as it would help them to estimate the amount used.

\section{Control group}

Children in the control group carried on with their usual health education lessons as in the curriculum, and these lessons did not contain information on salt.

\section{Outcome measures}

Our primary outcome was the difference between the intervention and the control group in the change of salt intake as measured by 24 hour urinary sodium from baseline to the end of the trial. The secondary outcome was the difference between the two groups in the change of blood pressure.

All outcome assessments were carried out at baseline and at the end of the trial in exactly the same way in all schools for all participants, irrespective of their assignment to intervention or control group.

We carried out two consecutive 24 hour urine collections. Trained research staff carefully instructed participants on how to accurately collect 24 hour urine samples. On the first visit to the participants' home, the researchers asked the participants to empty their bladder and discard the urine. The researchers recorded the start time and date of the 24 hour urine collection. They then gave the participants the collection equipment including containers and collection aids such as carrier bags. The participants were instructed to collect all subsequent urine voids over the next 24 hour period. On the second day at the same time, the researchers revisited the participants' home and asked them to pass the last urine into the container. The researchers recorded the finish time of the first 24 hour urine collection. The researchers then gave the participants the collection equipment for the second 24 hour urine collection and repeated the process. Participants were told to take spare urine containers with them when they went to school or work. Spare collection equipment was also available in the schools, in case children forgot to bring containers. For most families, collections were made on the same days of the week for baseline and follow-up. In the event that the participant missed one or more urine voids or spilt $>10 \%$ of the total 24 hour urine volume, the participant was asked to do a further 24 hour collection.

The urine samples were measured for volume and sodium, potassium, and creatinine concentrations. An ion selective electrode method was used for sodium and potassium analysis (AC9102 electrolyte analyzer, Audicom Medical Technology, Jiangsu) and Jaffe method for creatinine (Hitachi 7080 automatic biochemical analyzer, Japan). The biochemists who performed the urinary electrolyte measurements were not aware of the participants' group allocation.

We used the average of the two 24 hour urinary measurements at each time point in the analysis. In one child and six adults, however, we had only one 24 hour urine collection at baseline; and in one adult we had only one 24 hour urine collection at follow-up. In these cases, we used one 24 hour urinary measurement. 
Trained researchers measured blood pressure and pulse rate at the participants' homes using a validated automatic blood pressure monitor (Omron HEM-7301-IT, Amsterdam) with an appropriately sized cuff. After participants had rested for 10 minutes in a quiet room, three readings were taken in the right arm at two minute intervals with the participants in the sitting position and the arm supported at heart level. We used the average of the last two measurements for the analysis. Body weight and height were measured in participants without shoes or heavy clothes, with a standardised protocol. Both indoor and outdoor temperatures were measured with a thermometer (Anymetre, JR913).

\section{Project timeline}

The baseline assessments were carried out between late May and early July 2013-that is, before the school summer holiday. Randomisation took place during the summer holiday in August. The intervention programme was carried out during the school term from September to December. The follow-up assessments were carried out between late November and December 2013.

\section{Sample size calculation}

Based on a pilot study in schoolchildren aged 8-9 in London, in which salt intake measured by 24 hour urinary sodium was about $5 \mathrm{~g} /$ day with an SD of 2.15 and intracluster correlation coefficient of $0.01,22$ we estimated that a sample size of 240 children from 24 schools (cluster size 10) would provide $90 \%$ power to detect a difference of $20 \%$ in salt intake (that is, $1.0 \mathrm{~g} /$ day) between the two groups, with $\mathrm{a}=0.05$ and allowing for a $10 \%$ dropout rate. ${ }^{23}$ To ensure that the study had a sufficient power, we recruited 28 schools with 279 children.

We aimed to recruit two adults per family. We estimated that a sample size of 560 would provide a power of $>95 \%$ with $\alpha=0.05$ to detect a difference of $20 \%$ in salt intake (that is, $2 \mathrm{~g} /$ day reduction from $10 \mathrm{~g} /$ day), assuming an SD of 3.5. ${ }^{24}$

\section{Statistical analysis}

Our main analysis was based on intention to treat. We tested the effect of the intervention on the outcomes using linear mixed models with participants nested within family units and families nested within school units. We included group (intervention, control), time (baseline, end of trial), and timexgroup interaction, with the timexgroup interaction term indicating differential change by group from baseline to the end of the trial. To account for missing data on continuous outcomes, we used the likelihood based random effects model that uses all available data and provides valid estimates of the intervention effects when data are missing at random. We adjusted for the stratification variables at randomisation (school location and class size) and potential confounding variables including age (children $=1$; adults $\leq 40=2,40-60=3,>60=4)$, sex (male $=0$; female $=1$ ), body mass index (BMI), and indoor and outdoor temperature. Change over time was incorporated by inclusion of baseline values in the model.
We carried out various sensitivity analyses to examine the robustness of the conclusions of the primary analysis. We performed an analysis based on intention to treat but excluded possibly incomplete 24 hour urine collections defined as urine volume $<500 \mathrm{ml} / 24$ hour or creatinine $<4.0 \mathrm{mmol} / 24$ hour (women) or $<6.0 \mathrm{mmol} / 24$ hour (men) ${ }^{25}$ for adults and $<300 \mathrm{ml} / 24$ hour ${ }^{26}$ or creatinine $<5$ th centile (that is, $<2.5 \mathrm{mmol} / 24$ hour for girls and $<2.9 \mathrm{mmol} / 24$ hour for boys) for children. From a total of 3268 urine collections, we excluded 225. If one of the two 24 hour urine collections was incomplete, we used only one in the sensitivity analysis. We also carried out an analysis including only participants who completed both baseline and end trial assessments (named as "completers") and a per protocol analysis that included completers with complete 24 hour urine collections (that is, excluding possibly incomplete 24 hour urine collections).

We used SAS (version 9.4) for the analyses. Results are reported as mean, SD, SE, and 95\% confidence interval when appropriate. All analyses were two sided, and $\mathrm{P}<0.05$ was considered significant.

\section{Results}

We recruited 28 schools with 28 classes into the study. From 548 children who met the inclusion criteria for assessments, we randomly selected 357. From these children's families, we invited 713 adults. Of those recruited, 279 children and 553 adults completed the baseline assessments. During the trial, five children $(1.8 \%)$ and 21 adults (3.8\%) were lost to follow-up. Figure 1 shows the number of individuals by group and the reasons for loss to follow-up. There was no significant difference between the participants who were lost to follow-up and those who completed the study in age, sex, salt intake, or blood pressure in children or adults (data not shown). BMI, however, was higher in children who were lost to follow-up $(22.0 v 16.8, \mathrm{P}<0.001)$.

Table 1 shows the baseline characteristics of the participants. The mean age was 10.1 for children and 43.8 for adults. The two groups were well balanced in most parameters except for self reported hypertension, which was slightly higher in the intervention group.

In the intervention group the average amount of salt used as measured by weighing showed an immediate and large decrease within the first two weeks of the intervention. From week two to four, there was a small fluctuation, and then a gradual and sustained reduction throughout the whole duration of the study (fig 2).

Table 2 shows the primary and secondary outcomes and other 24 hour urinary measurements. For all participants together, the mean baseline salt intake, as calculated from 24 hour urinary sodium, was 9.8 (SE 0.3) g/day in the control group and 10.8 (SE 0.3) g/day in the intervention group. During the study, salt intake increased in the control group and decreased in the intervention group. Therefore, the mean effect comparing the intervention with the control group was $-2.6 \mathrm{~g} / \mathrm{day}$ (95\% confidence interval -3.2 to $-2.0 ; \mathrm{P}<0.001)$, representing a $26 \%$ reduction. Systolic and diastolic blood pressure showed an increase from baseline to the end of the trial 


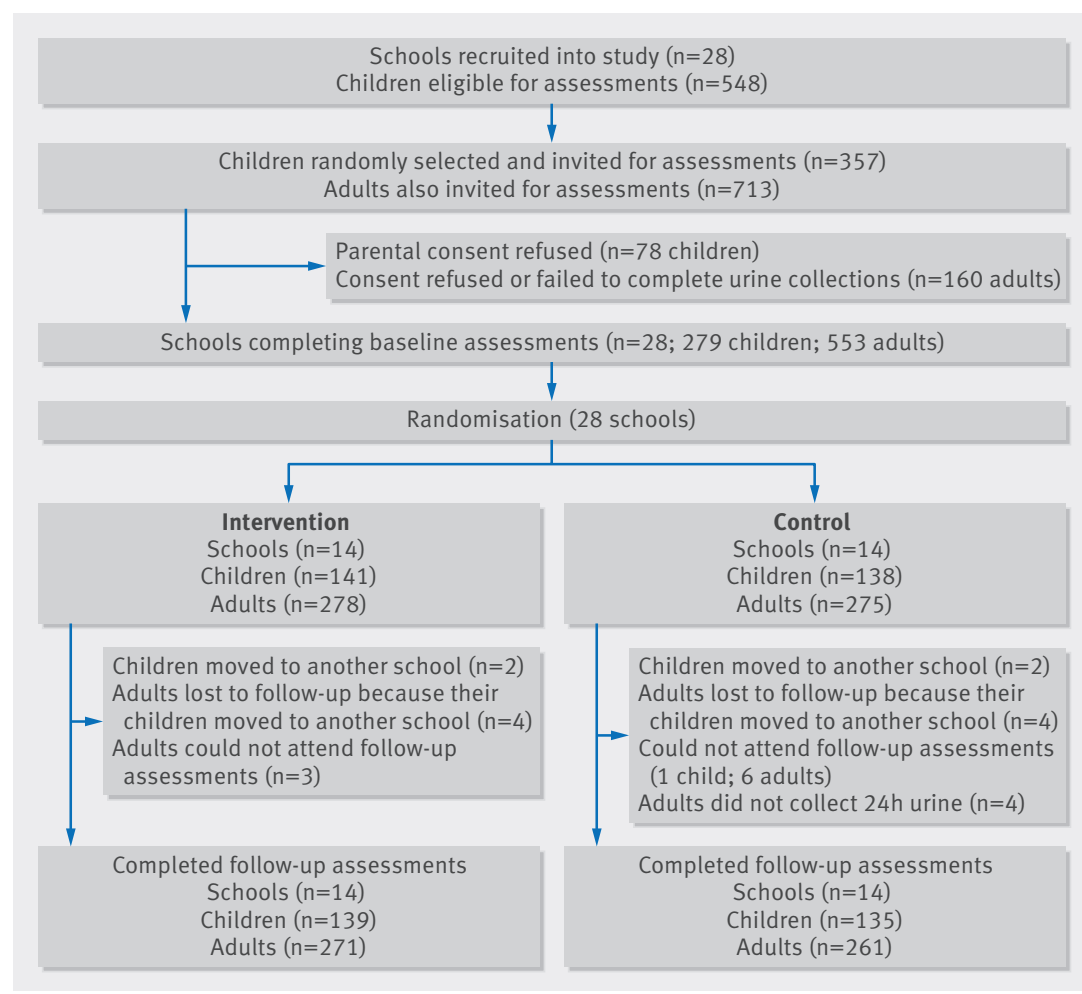

Fig 1 | Trial profile of school based education programme to reduce salt intake in children and their families interval -3.7 to $-2.2 \mathrm{~g} /$ day; $\mathrm{P}<0.001$ ), representing a $25 \%$ reduction compared with the control. The mean effect on systolic blood pressure was $-2.3 \mathrm{~mm} \mathrm{Hg}(-4.5$ to $-0.04 \mathrm{~mm} \mathrm{Hg}$ ), $\mathrm{P}<0.05)$. The effect on diastolic blood pressure was not significant.

Table 3 shows the results from sensitivity analyses. The first analysis excluded possibly incomplete 24 urine collections. As expected, the absolute levels of salt intake were higher than those when we included all 24 hour urine collections. The primary outcome, however-that is, the difference between the two groups in the change of salt intake-was similar to that from the main analysis. The results for completers and per protocol analyses were close to those from the corresponding analyses with all participants included.

From baseline to the end of the trial there was a large fall in ambient temperature. In the control group the mean outdoor temperature fell from $26.1^{\circ} \mathrm{C}$ to $2.3^{\circ} \mathrm{C}$ and the mean indoor temperature fell from $25.5^{\circ} \mathrm{C}$ to $15.8^{\circ} \mathrm{C}$; in the intervention group the figures were from $26.1^{\circ} \mathrm{C}$ to $1.1^{\circ} \mathrm{C}$ and from $25.5^{\circ} \mathrm{C}$ to $15.4^{\circ} \mathrm{C}$, respectively.

\section{Discussion}

The School-EduSalt trial is the first to explore the use of a primary school setting to educate children on salt reduction and to empower children to deliver the message to their families. On average, salt intake was reduced by $1.9 \mathrm{~g} /$ day (27\%) in children and $2.9 \mathrm{~g} /$ day (25\%) in adults over a period of one school term of about 3.5 months. This reduction in salt intake was accompanied by a significant fall in systolic blood pressure in adults. These findings show that the SchoolEduSalt approach is feasible and effective in lowering salt intake in both children and their families. difference in diastolic blood pressure was not significant (table 2).

In children, the mean baseline salt intake was 6.8 (SE 0.3) g/day in the control group and 7.3 (SE 0.3) g/day in the intervention group. The mean effect size after the intervention was $-1.9 \mathrm{~g} /$ day (95\% confidence interval -2.6 to $-1.3 \mathrm{~g} /$ day; $\mathrm{P}<0.001$ ), representing a $27 \%$ reduction compared with the control group. The effect on blood pressure was not significant (table 2).

In adults, the mean baseline salt intake was 11.3 (SE 0.4 ) g/day and 12.6 (SE 0.4) g/day for the control and intervention groups, respectively. The mean effect size after the intervention was $-2.9 \mathrm{~g} /$ day (95\% confidence

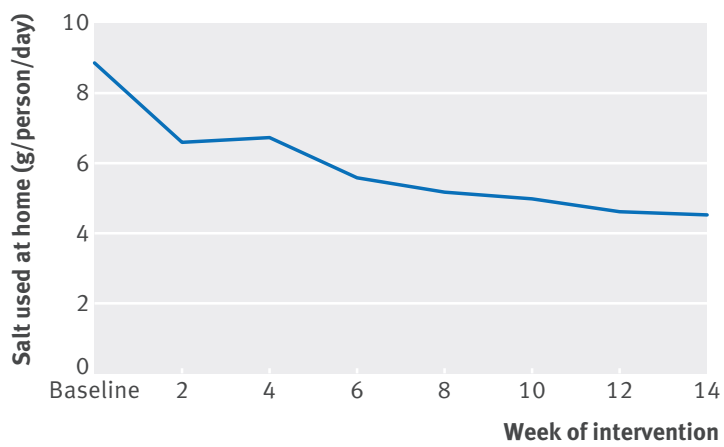

Fig $2 \mid$ Amount of salt used in intervention group according to weight of salt containers

\section{Salt intake}

We used repeat 24 hour urine collections (the most accurate method) to measure salt intake in a large number of primary school children in northern China and showed that the intake was high. A conservative estimate indicated that the average salt intake at baseline was $7.0 \mathrm{~g} /$ day for children aged 10, which is $40 \%$ higher than the WHO recommended level of $5 \mathrm{~g} /$ day for adults. We also found a high current salt intake in the adult family members, with an average baseline intake of $11.7 \mathrm{~g} /$ day, which is more than double the WHO recommended level.

We weighed the amount of salt used by the family every two weeks and showed a sustained reduction over the study period. Despite this, we focused on the urinary data because the dietary method is known to be unreliable in estimating salt intake-for example, some of the salt was used for soaking eggs rather than consumed. We collected two 24 hour urine samples at each time point, and the collections were carefully supervised with both the start and finish time recorded by trained research staff. We are sure that there was no over-collection, however, it is difficult to know whether there was any under-collection. Although the participants who admitted having missed urine voids were asked to do another 24 hour urine collection, it is still 


\begin{tabular}{|c|c|c|c|}
\hline & $\begin{array}{l}\text { Control (no salt } \\
\text { education) }\end{array}$ & $\begin{array}{l}\text { Intervention } \\
\text { (salt education) }\end{array}$ & All \\
\hline \multicolumn{4}{|l|}{ Cluster level } \\
\hline Schools & 14 & 14 & 28 \\
\hline Median (range) No of children/school & $10(9-10)$ & $10(10-11)$ & $10(9-11)$ \\
\hline Median (range) No of adults/school & $20(17-20)$ & $20(19-20)$ & $20(17-20)$ \\
\hline Median (range) No of adults/family & $2(1-2)$ & $2(1-2)$ & $2(1-2)$ \\
\hline \multicolumn{4}{|l|}{ Individual level } \\
\hline Total participants & 413 & 419 & 832 \\
\hline \multicolumn{4}{|l|}{ Children: } \\
\hline Total (No, \% boys) & $138(67,48.6)$ & $141(67,47.5)$ & $279(134,48.0)$ \\
\hline Mean (SD) age (year) & $10.2(0.5)$ & $10.0(0.5)$ & $10.1(0.5)$ \\
\hline Mean (SD) weight (kg) & $33.3(7.2)$ & $33.4(7.8)$ & $33.3(7.5)$ \\
\hline Mean (SD) height $(\mathrm{cm})$ & $140.7(6.6)$ & $139.2(6.2)$ & $140.0(6.5)$ \\
\hline Mean (SD) BMI & $16.7(2.7)$ & $17.1(3.2)$ & $16.9(3.0)$ \\
\hline \multicolumn{4}{|l|}{ Adults: } \\
\hline Total (No, \% men) & $275(133,48.4)$ & $278(135,48.6)$ & $553(268,48.5)$ \\
\hline Parents & $208(75.6)$ & $203(73.0)$ & $411(74.3)$ \\
\hline Grandparents & $67(24.4)$ & $75(27.0)$ & $142(25.7)$ \\
\hline Mean (SD) age (year) & $43.6(11.8)$ & $43.9(12.5)$ & $43.8(12.2)$ \\
\hline Mean (SD) weight (kg) & $66.2(12.9)$ & $66.1(11.6)$ & $66.2(12.3)$ \\
\hline Mean (SD) Height (cm) & $162.8(8.7)$ & $162.4(8.0)$ & $162.6(8.4)$ \\
\hline Mean (SD) BMI & $24.9(3.6)$ & $25.0(3.4)$ & $24.9(3.5)$ \\
\hline \multicolumn{4}{|l|}{ Education: } \\
\hline$\leq 6$ year & $55(20.0)$ & $59(21.2)$ & $114(20.6)$ \\
\hline $6-9$ year & $132(48.0)$ & $137(49.3)$ & $269(48.6)$ \\
\hline$>9$ year & $88(32.0)$ & $82(29.5)$ & $170(30.7)$ \\
\hline Alcohol drinkers & $101(36.7)$ & $84(30.2)$ & $185(33.5)$ \\
\hline Smokers & $79(28.7)$ & $76(27.3)$ & $155(28.0)$ \\
\hline Self reported hypertension & $33(12.0)$ & $42(15.1)$ & $75(13.6)$ \\
\hline BP treatment in hypertensives & $28(84.8)$ & $36(85.7)$ & $64(85.3)$ \\
\hline
\end{tabular}

possible that some participants did not report missing urine collection. Exclusion of potentially incomplete collections, as expected, led to a slightly higher mean salt intake for both baseline and the end of the trial and for both the intervention and control groups. It is therefore likely that our main results have underestimated the average salt intake of the study population. This is unlikely to alter the primary outcome-that is, the difference between the intervention and control group. Indeed, various sensitivity analyses showed consistent findings (table 3).

\section{Comparison with other studies}

Our baseline finding of a high salt intake in adults is consistent with the findings of several recent studies in northern China, where salt intake estimated from 24 hour urinary sodium, ranged from $11.8 \mathrm{~g} /$ day to 13.9 g/day. ${ }^{20} 2728$ The variations between studies could be attributable to differences in geographical regions, study populations, seasons when the study was carried out, and criteria used to assess over-collection and under-collection.

During our study, the control group showed an increase in salt intake. This is probably because of seasonal influence as our baseline assessments were carried out in summer and the follow-up was in winter. Salt intake in northern China is usually higher in winter because people eat more pickles because of reduced availability of fresh vegetables.

Many previous studies have reported seasonal variations in blood pressure. ${ }^{29}$ A large study involving over 500000 adults from 10 geographically diverse regions of China over a four year period showed a strong inverse association between blood pressure and outdoor temperature, with $5.7 \mathrm{~mm} \mathrm{Hg}$ higher systolic blood pressure per $10^{\circ} \mathrm{C}$ lower temperature. In our study, we observed a large fall in both indoor and outdoor temperature from baseline to the end of the trial. This could explain the increase in blood pressure observed in our study, though the rise in blood pressure was significantly smaller in the intervention group than in the control group, because of salt reduction.

Previous meta-analysis of randomised trials on salt reduction showed that a $1 \mathrm{~g} /$ day reduction in salt intake led to about a $1 \mathrm{~mm} \mathrm{Hg}$ reduction in systolic blood pressure. ${ }^{5}$ It has also been shown that, for a given reduction in salt intake, the fall in blood pressure was larger in older people than younger individuals and larger in those with than without hypertension. ${ }^{5}$ Our current study showed a $2.3 \mathrm{~mm} \mathrm{Hg}$ reduction in systolic blood pressure with a $2.9 \mathrm{~g} /$ day reduction in salt intake in adults. Given that our adult participants were relatively young, with a mean age of 44 , and predominately normotensive, it is therefore not surprising that the fall in systolic blood pressure was slightly smaller than that found in the meta-analysis of salt reduction trials. Our study also showed that in children, a reduction of $1.9 \mathrm{~g} / \mathrm{day}$ (27\%) in salt intake was related to a fall in blood pressure of $0.8 / 1.2 \mathrm{~mm} \mathrm{Hg}$. Although this is not significant, the magnitude of the fall in blood pressure is similar to that predicted from a meta-analysis of salt reduction trials in children. ${ }^{16}$

\section{Public health implications}

It is well established that blood pressure throughout its range starting from $115 / 75 \mathrm{~mm} \mathrm{Hg}$ is a major cause of cardiovascular disease.$^{30} \mathrm{~A}$ reduction in salt intake through its effect on blood pressure should reduce the risk of cardiovascular disease. Based on the meta-analysis of trials on treatment of blood pressure, ${ }^{31}$ we estimated that a $2.3 \mathrm{~mm} \mathrm{Hg}$ reduction in systolic blood pressure that occurred with salt reduction would reduce incidence of stroke by about $9 \%$ and ischaemic heart disease by about $5 \%$. In China this could prevent about 153000 deaths from strokes and about 47000 deaths from ischaemic heart disease a year.

Our findings have major public health implications. China, like many other developing countries, is experiencing fast epidemiological transition with a rapid increase in the disease burden attributed to blood pressure. ${ }^{32}$ Traditionally, prevention and control of hypertension have been managed within the healthcare system. ${ }^{33}$ In China and other developing countries, however, the health services are less developed with little capacity for prevention. The School-EduSalt offers a novel approach to preventing diseases related to raised blood pressure by reducing population salt intake. This approach has several advantages. Because 
Table 2 | Salt intake as calculated from 24 hour urinary sodium, other urinary measurements, and blood pressure based on intention to treat analysis in study of school based education programme to reduce salt intake in children and their families

\begin{tabular}{|c|c|c|c|c|c|c|c|}
\hline & \multicolumn{3}{|c|}{ Control (no salt education) } & \multicolumn{3}{|c|}{ Intervention (salt education) } & \multirow[b]{2}{*}{$\begin{array}{l}\text { Adjusted differencet (intervention } \\
v \text { control) }(95 \% \mathrm{CI}), \mathrm{P} \text { value }\end{array}$} \\
\hline & $\begin{array}{l}\text { Baseline* } \\
\text { (SE) }\end{array}$ & $\begin{array}{l}\text { End of } \\
\text { trial* }^{*}(\mathrm{SE})\end{array}$ & $\begin{array}{l}\text { Change from } \\
\text { baseline* }(95 \% \\
\text { CI) }\end{array}$ & $\begin{array}{l}\text { Baseline* } \\
\text { (SE) }\end{array}$ & $\begin{array}{l}\text { End of } \\
\text { trial* }^{*}(\mathrm{SE})\end{array}$ & 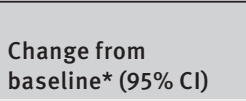 & \\
\hline \multicolumn{8}{|l|}{ All participants } \\
\hline Urine volume $(\mathrm{mL} / 24 \mathrm{~h})$ & $1337(47)$ & $1475(48)$ & 138 (89 to 188 ) & $1376(47)$ & $1411(47)$ & 35 (-14 to 83$)$ & $-99(-169$ to -30$), 0.005$ \\
\hline Urinary sodium (mmol/24h) & $167.6(5.7)$ & $183.1(5.7)$ & 15.5 (8.7 to 22.3) & $184.3(5.7)$ & $155.9(5.7)$ & $-28.5(-35.2$ to -21.8$)$ & $-44.4(-53.9$ to -34.9$),<0.001$ \\
\hline Salt (g/d) & $9.8(0.3)$ & $10.7(0.3)$ & $0.9(0.5$ to 1.3$)$ & $10.8(0.3)$ & $9.1(0.3)$ & $-1.7(-2.1$ to -1.3$)$ & $-2.6(-3.2$ to -2.0$),<0.001$ \\
\hline Urinary potassium (mmol/24h) & $32.5(0.9)$ & $33.9(0.9)$ & $1.4(-0.04$ to 2.8$)$ & $31.8(0.9)$ & $33.7(0.9)$ & 2.0 (0.6 to 3.3) & $0.6(-1.4$ to 2.5$), 0.59$ \\
\hline Urinary creatinine (mmol/24h) & $7.8(0.2)$ & $7.8(0.2)$ & $0.1(-0.1$ to 0.2$)$ & $7.9(0.2)$ & $7.8(0.2)$ & $-0.1(-0.3$ to 0.03$)$ & $-0.2(-0.5$ to 0.04$), 0.10$ \\
\hline Systolic BP (mm Hg) & $118.1(1.2)$ & $122.9(1.2)$ & 4.8 (3.6 to 6.0$)$ & $120.1(1.2)$ & $123.6(1.2)$ & $3.5(2.3$ to 4.7$)$ & $-1.8(-3.4$ to -0.1$),<0.04$ \\
\hline Diastolic BP (mm Hg) & $75.5(0.9)$ & $78.8(1.0)$ & $3.3(2.3$ to 4.4$)$ & $76.5(0.9)$ & $79.1(0.9)$ & $2.6(1.6$ to 3.6$)$ & $-1.1(-2.5$ to 0.4$), 0.15$ \\
\hline \multicolumn{8}{|l|}{ Children } \\
\hline Urine volume $(\mathrm{mL} / 24 \mathrm{~h})$ & $862(38)$ & $952(38)$ & $90(35$ to 145$)$ & $867(37)$ & $831(38)$ & $-37(-91$ to 18$)$ & $-124(-201$ to -46$), 0.002$ \\
\hline Urinary sodium (mmol/24h) & $116.7(5.2)$ & $137.2(5.2)$ & $\begin{array}{l}20.5(12.6 \text { to } \\
28.4)\end{array}$ & $124.2(5.1)$ & $112.2(5.1)$ & $-12.1(-19.9$ to -4.2$)$ & $-33.3(-44.2$ to -22.3$),<0.001$ \\
\hline Salt (g/d) & $6.8(0.3)$ & $8.0(0.3)$ & $1.2(0.7$ to 1.7$)$ & $7.3(0.3)$ & $6.6(0.3)$ & $-0.7(-1.2$ to -0.2$)$ & $-1.9(-2.6$ to -1.3$),<0.001$ \\
\hline Urinary potassium (mmol/24h) & $25.4(0.9)$ & $25.9(0.9)$ & $0.5(-1.2$ to 2.3$)$ & $23.5(0.9)$ & $25.3(0.9)$ & $1.8(0.1$ to 3.5$)$ & 1.3 (-1.1 to 3.8$), 0.28$ \\
\hline Urinary creatinine (mmol/24h) & $4.9(0.2)$ & $5.2(0.2)$ & $0.4(0.2$ to 0.5$)$ & $4.7(0.2)$ & $5.1(0.2)$ & $0.4(0.2$ to 0.5$)$ & $-0.006(-0.2$ to 0.2$), 0.96$ \\
\hline Systolic BP (mm Hg) & $106.2(1.0)$ & $110.6(1.0)$ & $4.4(2.8$ to 6.0$)$ & $106.2(1.0)$ & $110.0(1.0)$ & $3.8(2.3$ to 5.4$)$ & $-0.8(-3.0$ to 1.5$), 0.51$ \\
\hline Diastolic BP (mm Hg) & $66.8(1.1)$ & $70.2(1.1)$ & $3.4(1.6$ to 5.1$)$ & $67.0(1.1)$ & $69.4(1.1)$ & $2.4(0.7$ to 4.1$)$ & $-1.2(-3.7$ to 1.2$), 0.33$ \\
\hline \multicolumn{8}{|l|}{ Adults } \\
\hline Urine volume $(\mathrm{mL} / 24 \mathrm{~h})$ & $1577(61)$ & $1740(62)$ & 163 (94 to 232) & $1636(61)$ & $1709(61)$ & 73 (5 to 141$)$ & $-86(-183$ to 10$), 0.08$ \\
\hline Urinary sodium (mmol/24h) & $193.4(6.7)$ & $206.6(6.8)$ & 13.2 (3.8 to 22.6) & $215.1(6.7)$ & $178.5(6.7)$ & $-36.6(-45.8$ to -27.3$)$ & $-50.0(-63.1$ to -36.8$),<0.001$ \\
\hline Salt $(g / d)$ & $11.3(0.4)$ & $12.1(0.4)$ & $0.8(0.2$ to 1.3$)$ & $12.6(0.4)$ & $10.4(0.4)$ & $-2.1(-2.7$ to -1.6$)$ & $-2.9(-3.7$ to -2.2$),<0.001$ \\
\hline Urinary potassium (mmol/24h) & $36.1(1.0)$ & $38.0(1.0)$ & $1.9(-0.1$ to 3.8$)$ & $36.0(1.0)$ & $38.1(1.0)$ & $2.1(0.2$ to 4.0$)$ & 0.1 (-2.6 to 2.9$), 0.93$ \\
\hline Urinary creatinine (mmol/24h) & $9.3(0.2)$ & $9.2(0.2)$ & $-0.1(-0.4$ to 0.1$)$ & $9.5(0.2)$ & $9.1(0.2)$ & $-0.4(-0.7$ to -0.2$)$ & $-0.3(-0.6$ to 0.04$), 0.09$ \\
\hline Systolic BP $(\mathrm{mm} \mathrm{Hg})$ & $124.1(1.5)$ & $129.1(1.5)$ & $5.0(3.4$ to 6.6$)$ & $127.1(1.5)$ & $130.5(1.5)$ & $3.4(1.8$ to 4.9$)$ & $-2.3(-4.5$ to -0.04$),<0.05$ \\
\hline Diastolic BP (mm Hg) & $79.9(1.0)$ & $83.2(1.1)$ & $3.3(2.0$ to 4.6$)$ & $81.4(1.0)$ & $84.1(1.0)$ & 2.8 (1.5 to 4.0$)$ & $-0.9(-2.8$ to 0.9$), 0.31$ \\
\hline
\end{tabular}

$\mathrm{BP}=$ blood pressure.

*Adjusted for stratification variables at randomisation (school location and class size).

TAdjusted for age, sex, BMI, stratification variables at randomisation (school location and class size), and indoor and outdoor temperature.

universal primary education is a common goal in most national education policies, the School-EduSalt approach is likely to have a greater impact on preventing hypertension than the traditional way of using the healthcare system. Conveying a salt reduction message to children has the potential to set habits and attitudes that will persist throughout adulthood. Furthermore, the School-EduSalt approach reaches a wide range of the population from children to adults and therefore has the potential to achieve a large impact.

It is important to emphasise that to achieve the greatest reduction in population salt intake, the SchoolEduSalt approach should be combined with other strategies, which were not included in our study. For example, in children who have meals in schools, a reduction in the salt content will help reduce children's

Table 3 | Sensitivity analysis for salt intake (g/day) as calculated from 24 hour urinary sodium

\begin{tabular}{|c|c|c|c|c|c|c|}
\hline & \multirow[b]{2}{*}{$\begin{array}{l}\text { No in control/ } \\
\text { intervention }\end{array}$} & \multicolumn{2}{|l|}{ Control } & \multicolumn{2}{|l|}{ Intervention } & \multirow{2}{*}{$\begin{array}{l}\text { Adjusted differencet } \\
\text { (intervention } v \text { control) } \\
(95 \% \text { Cl), P value }\end{array}$} \\
\hline & & Baseline* (SE) & $\begin{array}{l}\text { Change from baseline* } \\
(95 \% \mathrm{Cl})\end{array}$ & Baseline* (SE) & $\begin{array}{l}\text { Change from baseline* } \\
(95 \% \mathrm{Cl})\end{array}$ & \\
\hline \multicolumn{7}{|c|}{ Population excluding possible incomplete $24 \mathrm{~h}$ urine: } \\
\hline All & $411 / 415$ & $10.0(0.3)$ & 0.9 (0.6 to 1.3$)$ & $11.0(0.3)$ & $-1.6(-2.0$ to -1.2$)$ & $-2.6(-3.1$ to -2.1$),<0.001$ \\
\hline Children & $138 / 140$ & $7.0(0.3)$ & $1.2(0.7$ to 1.6$)$ & $7.5(0.3)$ & $-0.7(-1.1$ to -0.2$)$ & $-1.9(-2.6$ to -1.2$),<0.001$ \\
\hline Adults & $273 / 275$ & $11.6(0.4)$ & $0.8(0.3$ to 1.4$)$ & $12.8(0.4)$ & $-2.1(-2.6$ to -1.6$)$ & $-3.0(-3.7$ to -2.2$),<0.001$ \\
\hline \multicolumn{7}{|c|}{ Completers: $\ddagger$} \\
\hline All & $396 / 410$ & $9.8(0.3)$ & $0.9(0.5$ to 1.3$)$ & $10.8(0.3)$ & $-1.7(-2.1$ to -1.3$)$ & $-2.6(-3.2$ to -2.1$),<0.001$ \\
\hline Children & $135 / 139$ & $6.8(0.3)$ & $1.2(0.8$ to 1.7$)$ & $7.2(0.3)$ & $-0.7(-1.2$ to -0.2$)$ & $-1.9(-2.6$ to -1.3$),<0.001$ \\
\hline Adults & $261 / 271$ & $11.4(0.4)$ & $0.8(0.2$ to 1.3$)$ & $12.7(0.4)$ & $-2.2(-2.7$ to -1.6$)$ & $-3.0(-3.7$ to -2.2$),<0.001$ \\
\hline \multicolumn{7}{|c|}{ Per protocol population:§ } \\
\hline All & $381 / 393$ & $10.0(0.3)$ & $1.0(0.6$ to 1.4$)$ & $11.0(0.3)$ & $-1.6(-2.0$ to -1.2$)$ & $-2.6(-3.2$ to -2.1$),<0.001$ \\
\hline Children & $132 / 137$ & $7.0(0.3)$ & $1.2(0.7$ to 1.7$)$ & $7.5(0.3)$ & $-0.7(-1.1$ to -0.2$)$ & $-1.9(-2.6$ to -1.3$),<0.001$ \\
\hline Adults & $249 / 256$ & $11.6(0.4)$ & $0.9(0.3$ to 1.4$)$ & $12.9(0.4)$ & $-2.1(-2.6$ to -1.6$)$ & $-3.0(-3.7$ to -2.3$),<0.001$ \\
\hline
\end{tabular}

*Adjusted for stratification variables at randomisation (school location and class size).

tAdjusted for age, sex, body mass index, stratification variables at randomisation (school location and class size), and indoor and outdoor temperature.

¥Participants with 24 hour urine collections at baseline and end of trial.

$\S$ Completers with complete 24 hour urine collections. 
salt intake further. Additionally, there has been an increasing trend in the consumption of processed foods in China. ${ }^{34}$ It is therefore also important to work with the food industry to gradually reduce the amount of salt added to all processed foods, as is occurring successfully in the United Kingdom, with an accompanying fall in population blood pressure and mortality from cardiovascular disease. ${ }^{835}$

\section{Conclusions}

Our study shows that, in northern China, salt intake as measured by 24 hour urinary sodium is high in both children and adults. An education programme targeted at primary school children is effective in lowering salt intake in children and their families. These results should be broadly applicable to most schools in China as our intervention programme was delivered using the usual health education lessons as in the national school curriculum. A nationwide implementation of the education programme could have a large impact on reducing population salt intake in China and morbidity and mortality from cardiovascular disease.

We thank Peter Sever, Francesco Cappuccio, Kiang Liu, and Dong Zhao for their support and advice; the Changzhi Education Bureau, the headteachers and teachers of all participating schools, and children and their families who were involved in the research; all members of the field research team, particularly Yanbo Han, Peifen Duan, Zhifang Li, Jianbing Zhang, Cailing Wei, Yanli Zhai, Ruikai Zhu, Hui Yang, and Xiaoyun Wang; Yide Yang, Xiaorui Shang, and Wenyi Niu for their help with the development of the education materials; and Elizabeth L Turner and Xian Li for their helpful advice on the statistical analyses.

Trial Steering Committee: Peter Sever (chair), Francesco Cappuccio, Kiang Liu, Dong Zhao, Feng He, Yangfeng Wu, and Graham MacGregor.

Contributors: FJH, YW, CN, and GAM designed the study. FJH and YW contributed equally to the project. JM and HW designed the education materials. XXF, JZ, YM, and JY contributed to data collection and implementation of the education programme. FJH and YM developed the analysis plan, performed statistical analyses, and took responsibility for the integrity of the data and the accuracy of the data analysis. FJH wrote the manuscript. All authors contributed to the revision and approved the final manuscript. FJH is guarantor.

Funding: The study was funded by the UK Medical Research Council (MR/J015903/1). The study is part of the GACD (Global Alliance for Chronic Disease) Hypertension Programme. The funder of the study had no role in the design of the study; the collection, analysis, and interpretation of the data; the writing of the manuscript; and the decision to submit the article for publication.

Competing interests: All authors have completed the ICMJE uniform disclosure form at www.icmje.org/coi disclosure.pdf (available on request from the corresponding author) and declare: $\mathrm{FJH}$ is a member of Consensus Action on Salt and Health (CASH) and World Action on Salt and Health (WASH). Both CASH and WASH are non-profit charitable organisations and FJH does not receive any financial support from CASH or WASH. GAM is chairman of Blood Pressure UK (BPUK), chairman of CASH, WASH, and Action on Sugar. BPUK, CASH, WASH, and Action on Sugar are non-profit charitable organisations. GAM does not receive any financial support from any of these organisations. CN is a member of WASH and AWASH (Australian division of World Action on Salt and Health) and does not receive any financial support from these organisations. $\mathrm{CN}$ has received remuneration from Meat and Livestock Australia, Nestle Health Science, and Dairy Health Consortium. These payments are unrelated to the submitted work. YM was sponsored by the China Scholarship Council while she was carrying out statistical analysis for this study at the Wolfson Institute of Preventive Medicine, Queen Mary University of London.

Ethical approval: The study protocol was approved by Queen Mary (University of London) research ethics committee (QMREC2012/81) and Peking University Health Science Centre IRB (IRB00001052-12072). Permissions were obtained from the local education authority (Changzhi
Education Bureau) and headteachers of the schools. All participants who took part in the assessments gave written informed consent. For children, participant assent and parental written consent were obtained. Transparency declaration: FJH affirms that the manuscript is an honest, accurate, and transparent account of the study being reported; that no important aspects of the study have been omitted; and that any discrepancies from the study as planned (and, if relevant, registered) have been explained.

Data sharing: No additional data available.

This is an Open Access article distributed in accordance with the Creative Commons Attribution Non Commercial (CC BY-NC 4.0) license, which permits others to distribute, remix, adapt, build upon this work non-commercially, and license their derivative works on different terms, provided the original work is properly cited and the use is non-commercial. See: http://creativecommons.org/licenses/ by-nc/4.0/.

1 Lawes CM, Vander Hoorn S, Rodgers A. Global burden of bloodpressure-related disease, 2001. Lancet 2008;371:1513-8.

2 World Health Report 2002: Reducing risks, promoting healthy life. WHO, 2002. www.who.int/whr/2002

3 Intersalt Cooperative Research Group. Intersalt: an international study of electrolyte excretion and blood pressure. Results for 24 hour urinary sodium and potassium excretion. BMJ 1988;297:319-28.

4 He FJ, MacGregor GA. Reducing population salt intake worldwide: from evidence to implementation. Prog Cardiovasc Dis 2010;52:363-82.

5 He FJ, Li J, MacGregor GA. Effect of longer term modest salt reduction on blood pressure: Cochrane systematic review and meta-analysis of randomised trials. BMJ 2013;346:f1325.

6 Aburto NJ, Ziolkovska A, Hooper L, Elliott P, Cappuccio FP, Meerpohl JJ. Effect of lower sodium intake on health: systematic review and meta-analyses. BM/ 2013:346:f1326.

7 He FJ, MacGregor GA. Salt reduction lowers cardiovascular risk: meta-analysis of outcome trials. Lancet 2011;378:380-2.

8 He FJ, Pombo-Rodrigues S, MacGregor GA. Salt reduction in England from 2003 to 2011: its relationship to blood pressure, stroke and ischaemic heart disease mortality. BMJ Open 2014;4:e004549.

9 Asaria P, Chisholm D, Mathers C, Ezzati M, Beaglehole R. Chronic disease prevention: health effects and financial costs of strategies to reduce salt intake and control tobacco use. Lancet 2007:370:2044-53.

10 Bibbins-Domingo K, Chertow GM, Coxson PG, Moran A, Lightwood IM, Pletcher MJ, et al. Projected effect of dietary salt reductions on future cardiovascular disease. N Engl J Med 2010;362:590-9.

11 National Institute for Health and Clinical Excellence. Guidance on the prevention of cardiovascular disease at the population level. NICE, 2010.http://guidance.nice.org.uk/PH25

12 First global ministerial conference on healthy lifestyles and noncommunicable disease control, 28-29 April 2011, Moscow. www. who.int/nmh/events/moscow_ncds_2011/en/

13 Beaglehole R, Bonita R, Horton R, Adams C, Alleyne G, Asaria P, et al. Priority actions for the non-communicable disease crisis. Lancet 2011;377:1438-47.

14 Lauer RM, Clarke WR. Childhood risk factors for high adult blood pressure: the Muscatine Study. Pediatrics 1989;84:633-41.

15 Chen $X$, Wang Y. Tracking of blood pressure from childhood to adulthood: a systematic review and meta-regression analysis. Circulation 2008;117:3171-80

16 He Fl, MacGregor GA. Importance of salt in determining blood pressure in children: meta-analysis of controlled trials. Hypertension 2006;48:861-9.

17 Wu Y, Huxley R, Li L, Anna V, Xie G, Yao C, et al. Prevalence, awareness, treatment, and control of hypertension in China: data from the China National Nutrition and Health Survey 2002. Circulation 2008:118:2679-86.

18 Zhao L, Stamler J, Yan LL, Zhou B, Wu Y, Liu K, et al. Blood pressure differences between northern and southern Chinese: role of dietary factors: the International Study on Macronutrients and Blood Pressure. Hypertension 2004:43:1332-7.

19 Anderson CA, Appel LJ, Okuda N, Brown IJ, Chan Q, Zhao L, et al. Dietary sources of sodium in China, Japan, the United Kingdom, and the United States, women and men aged 40 to 59 years: the INTERMAP study. J Am DietAssoc 2010;110:736-45.

20 Xu J, Wang M, Chen Y, Zhen B, Li J, Luan W, et al. Estimation of salt intake by 24-hour urinary sodium excretion: a cross-sectional study in Yantai, China. BMC Public Health 2014:14:136.

21 He FJ, Wu Y, Ma J, Feng X, Wang H, Zhang J, et al. A school-based education programme to reduce salt intake in children and their families (School-EduSalt): protocol of a cluster randomised controlled trial. BMJ Open 2013;3:pii:e003388.

22 Marrero NM, He FJ, Whincup P, Macgregor GA. Salt intake of children and adolescents in South London: consumption levels and dietary sources. Hypertension 2014;63:1026-32. 
23 Wu Y, Cai R, Zhou B, Xu X. Effects of genetic factors and dietary electrolytes on blood pressure of rural secondary school students in Hanzhong. Chin Med Sci 1991;6:148-52.

24 He FJ, Marciniak M, Visagie E, Markandu ND, Anand V, Dalton RN, et al. Effect of modest salt reduction on blood pressure, urinary albumin, and pulse wave velocity in white, black, and Asian mild hypertensives. Hypertension 2009;54:482-8.

25 Li N, Yan LL, Niu W, Labarthe D, Feng X, Shi J, et al. A large-scale cluster randomized trial to determine the effects of community-based dietary sodium reduction-the China Rural Health Initiative Sodium Reduction Study. Am Heart J 2013;166:815-22.

26 Maldonado-Martin A, Garcia-Matarin L, Gil-Extremera B, AvivarOyonarte C, Garcia-Granados ME, Gil-Garcia F, et al. Blood pressure and urinary excretion of electrolytes in Spanish schoolchildren. J Hum Hypertens 2002;16:473-8

27 Chen J, Tian Y, Liao Y, Yang S, Li Z, He C, et al. Salt-restriction-spoon improved the salt intake among residents in China. PLoS One 2013;8:e78963.

29 Zhang JY, Yan LX, Tang JL, Ma JX, Guo XL, Zhao WH, et al. Estimating daily salt intake based on $24 \mathrm{~h}$ urinary sodium excretion in adults aged 18-69 years in Shandong, China. BMJ Open 2014;4:e005089.

29 Lewington S, Li L, Sherliker P, Guo Y, Millwood I, Bian Z, et al. Seasonal variation in blood pressure and its relationship with outdoo temperature in 10 diverse regions of China: the China Kadoorie Biobank. J Hypertens 2012;30:1383-91.
30 Lewington S, Clarke R, Qizilbash N, Peto R, Collins R. Age-specific relevance of usual blood pressure to vascular mortality: a meta-analysis of individual data for one million adults in 61 prospective studies. Lancet 2002;360:1903-193.

31 Law MR, Morris JK, Wald NJ. Use of blood pressure lowering drugs in the prevention of cardiovascular disease: meta-analysis of 147 randomised trials in the context of expectations from prospective epidemiological studies. BMJ 2009;338:b1665.

32 Yang G, Wang Y, Zeng Y, Gao GF, Liang X, Zhou M, et al. Rapid health transition in China, 1990-2010: findings from the Global Burden of Disease Study 2010. Lancet 2013;381:1987-2015.

33 Liu Q, Wang B, Kong Y, Cheng KK. China's primary health-care reform. Lancet 2011;377:2064-6.

34 Du S, Batis C, Wang H, Zhang B, Zhang J, Popkin BM. Understanding the patterns and trends of sodium intake, potassium intake, and sodium to potassium ratio and their effect on hypertension in China. Am I Clin Nutr 2014:99:334-43.

35 He FJ, Brinsden HC, MacGregor GA. Salt reduction in the United Kingdom: a successful experiment in public health. J Hum Hypertens 2014;28:345-52.

(C) BMJ Publishing Group Ltd 2015

Appendix: Salt reduction education programme 